\title{
Differentially expressed kinase genes associated with trypsinogen activation in rat pancreatic acinar cells treated with taurolithocholic acid 3-sulfate
}

\author{
BIAO MA ${ }^{1}$, LIN WU $^{1}$, MING LU ${ }^{2}$, BO GAO $^{1}$, XIN QIAO $^{2}$, BEI SUN $^{1}$, DONGBO XUE $^{1}$ and WEIHUI ZHANG \\ ${ }^{1}$ Department of General Surgery, The First Affiliated Hospital of Harbin Medical University, \\ Harbin, Heilongjiang 150001, P.R. China; ${ }^{2}$ Department of Surgery, David Geffen School of Medicine, \\ University of Califonia at Los Angeles, Los Angeles, CA 90095, USA
}

Received November 25, 2012; Accepted February 27, 2013

DOI: $10.3892 / \mathrm{mmr} .2013 .1355$

\begin{abstract}
Trypsinogen activation is the initial factor involved in the development of all types of acute pancreatitis (AP) and has been suggested to be regulated by protein kinases. In the present study, AR42J rat pancreatic acinar cells were treated with taurolithocholic acid 3-sulfate (TLC-S), and trypsinogen activation was detected with bis-(CBZ-L-isoleucyl-L-prolyl-L-arginine amide) dihydrochloride (BZiPAR) staining and flow cytometry. Differentially expressed protein kinase genes were screened by Gene Chip analysis, and the functions of these kinases were analyzed. A significantly increased activation of trypsinogen in AR42J cells following treatment with TLC-S was observed. A total of 22 differentially expressed protein kinase genes were found in the TLC-S group, among which 19 genes were upregulated and 3 were downregulated. Based on the Kyoto Encyclopedia of Genes and Genomes (KEGG) database, kinase genes of the same KEGG pathways were connected to create a network through signaling pathways, and 10 nodes of kinases were identified, which were mitogen-activated protein kinase (Mapk)8, Mapk14, Map2k4, interleukin-1 receptor-associated kinase 3 (Irak3), ribosomal protein S6 kinase, $90 \mathrm{kDa}$, polypeptide 2 (Rps6ka2), protein kinase C, alpha (Prkca), v-yes-1 Yamaguchi sarcoma viral related oncogene homolog (Lyn), protein tyrosine kinase 2 beta (Ptk2b), p21 protein (Cdc42/Rac)activated kinase 4 (Pak4) and FYN oncogene related to SRC, FGR, YES (Fyn). The interactions between signaling pathways were further analyzed and a network was created. MAPK and calcium signaling pathways were found to be located at the
\end{abstract}

Correspondence to: Professor Dongbo Xue or Professor Weihui Zhang, Department of General Surgery, The First Affiliated Hospital of Harbin Medical University, 23 Youzheng Street, Harbin, Heilongjiang 150001, P.R. China

E-mail: xue9971@sina.com

E-mail: zhangweihui626@hotmail.com

Key words: acute pancreatitis, protein kinase, trypsinogen activation, signaling pathway center of the network. Thus, protein kinases constitute potential drug targets for AP treatment.

\section{Introduction}

During the development of acute pancreatitis (AP), trypsinogen in pancreatic acinar cells is aberrantly activated by external stimuli; the activated trypsin then activates additional digestive zymogens, leading to the damage and self-digestion of pancreatic acinar cells. Subsequently, during inflammatory cell infiltration, a large number of inflammatory molecules enter the circulatory system, causing systemic inflammatory response syndrome (SIRS), multiple organ dysfunction syndrome (MODS) and even death.

During this process, the abnormality of calcium concentration and protein kinase, the reduction in adenosine triphosphate (ATP) levels, the oxidative stress, as well as other reactions in pancreatic acinar cells contribute to the development of AP. All of these mechanisms are based on protein-kinasemediated intracellular signal transduction; thus, research on AP treatment involving the targeting protein kinases may prove useful for the treatment of multiple pathogeneses and may systematically inhibit the development of AP.

In acute biliary pancreatitis, pancreatic enzyme activation is caused by bile or some of its components, as well as other causes. Bile acid directly acts on pancreatic acinar cells by activating phosphatidylinositol 3-kinase (PI3K) to pathologically increase the calcium level and activate digestive zymogens, cell injury/death and inflammation pathways in cells (1). Bile acid also irritates inositol 1,4,5-trisphosphate (IP3) and ryanodine receptors, releasing the calcium stored in the endoplasmic reticulum and zymogen granules of acinar cells (2), thus leading to pathological calcium transients in cells. In 2010, Perides et al (3) demonstrated that taurolithocholic acid 3-sulfate (TLC-S) causes an alteration in the calcium concentration in cells by acting on the acinar cell surface (Gpbar 1), and they concluded that biliary AP may be a receptor-mediated disease.

The aim of this study was to provide evidence for the development of AP treatment strategies targeting protein kinases. Consequently, pancreatic acinar AR42J cells were treated with 
TLC-S. Trypsinogen activation in pancreatic acinar cells was observed, the differentially expressed protein kinase genes were screened by gene chip analysis, and the functions of these kinases were analyzed.

\section{Materials and methods}

Cell culture and processing. The AR42J rat pancreatic acinar cell line was purchased from the China Center for Type Culture Collection (CCTCC; Wuhan, China) and cultured in Ham's F12 medium (F12K; Invitrogen, Carlsbad, CA, USA), supplemented with $10 \%$ fetal bovine serum (FBS) (HyClone, Logan, UT, USA), $100 \mathrm{U} / \mathrm{ml}$ penicillin and $100 \mu \mathrm{g} / \mathrm{ml}$ streptomycin (Sigma, St. Louis, MO, USA). The cells were cultivated in an incubator containing $5 \% \mathrm{CO}_{2}$ at $37^{\circ} \mathrm{C}$. The activation of trypsinogen and the change in the proteome were assessed 20 min following TLC-S (Sigma) treatment.

Assessment of trypsinogen activation. Acinar cells were cultured for $20 \mathrm{~min}$ in HBS-EDTA (5 mM HEPES, $0.15 \mathrm{M}$ $\mathrm{NaCl}, 2 \mathrm{mM}$ EDTA; $\mathrm{pH}$ 7.35) with $5 \mathrm{mM}$ rhodamine 110 , bis-(CBZ-L-isoleucyl-L-prolyl-L-arginine amide) dihydrochloride (BZiPAR; Molecular Probes ${ }^{\circledR}$; Invitrogen), with the cell concentration adjusted to $10^{5}$ cells $/ \mathrm{ml}$. Trypsinogen activation was then assessed using a flow cytometer (FACSDiva, version 6.1; BD Biosciences, San Jose, NJ, USA) at an excitation wavelength of $485 \mathrm{~nm}$ following treatment with $200 \mu \mathrm{M} / 1$ TLC-S for an additional $20 \mathrm{~min}$. The experiment was performed in triplicate.

Detection of gene expression using DNA microarrays. The cells were collected, and total RNA was extracted using TRIzol reagent (Invitrogen) according to the manufacturer's instructions. Gene expression analyses were performed with the Rat 12x135K Gene Expression Array (NimbleGen Systems, Inc., Madison, WI, USA). Preparation of cDNA from $5 \mu \mathrm{g}$ of total RNA, hybridizations, washes and detection were performed in accordance to the NimbleGen gene expression analysis protocol (NimbleGen Systems, Inc.); the slides were scanned using the Axon GenePix 4000B microarray scanner.

Data analysis. Scanned images (TIFF format) were then imported into NimbleScan software (version 2.5) for grid alignment and expression data analysis. Expression data were normalized through quantile normalization and the Robust Multichip Average (RMA) algorithm included in the NimbleScan software. The Probe level (*_norm_RMA. pair) files and Gene level (*_RMA.calls) files were generated following normalization. All the gene level files were imported into GeneSpring GX software (version 11.5.1; Agilent Technologies, Inc., Santa Clara, CA, USA) for further analysis. Differentially expressed genes were identified through fold change filtering.

Based on the latest database of protein kinase (http:// kinasource.co.uk/Database/substrates.html), differentially expressed kinase genes were selected. The differentially expressed kinases genes which were singled out were annotated in the Kyoto Encyclopedia of Genes and Genomes (KEGG; http://www.kegg.jp) rat pathway. A visualized network was drawn with the software cytoscape (version 2.6.3) to exhibit
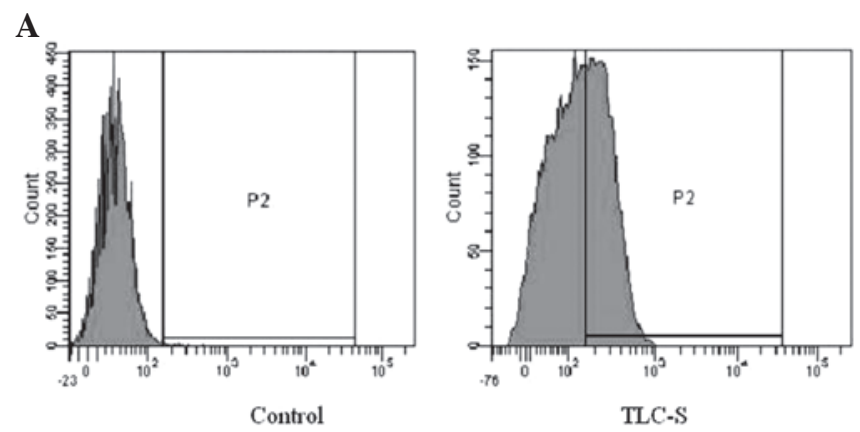

B

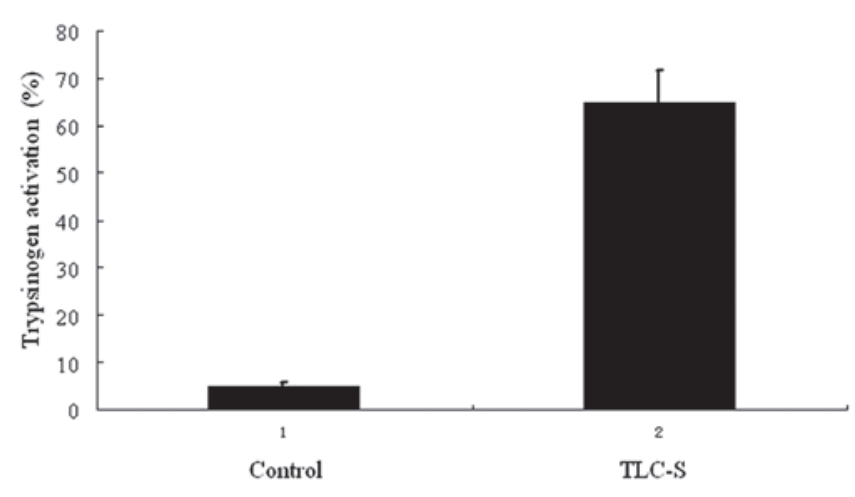

Figure 1. Trypsinogen activation in AR42J cells treated with taurolithocholic acid 3-sulfate (TLC-S). (A) Histogram of flow cytometry; (B) statistical chart. AR42J cells were stained with BZiPAR; BZiPAR was enzymatically lysed by activated trypsinogen (trypsin) and emited green fluorescence excited by an argon laser at $488 \mathrm{~nm}$, which was detected using a flow cytometer. Increased activation of trypsinogen was observed following treatment with TLC-S. The results are expressed as the means $\pm \mathrm{SD}(\mathrm{n}=3) . \mathrm{P}<0.05$.

the correlations between the kinases. Finally, each signaling pathway that annotated the differentially expressed kinase genes was imported into KEGG to search all related pathways, and the correlation was exhibited with a visualized network using software cytoscape (version 2.6.3).

\section{Results}

Trypsinogen activation. BZiPAR coupled with fluorescein rhodamine 110 , can not be excited to emit fluorescence prior to enzyme cleavage. However, following trypsin enzymatic lysis, rhodamine 110 emits green fluorescence excited by argon laser at $488 \mathrm{~nm}$. As a result, the degree of trypsinogen activation can be determined by detecting the fluorescence intensity. In this experiment, there were only trace amounts of activated trypsinogen found in the control group, while a significantly higher level was detected by flow cytometry following $20 \mathrm{~min}$ of treatment with TLC-S in the AR42J cells (Fig. 1).

Identification of differentially expressed protein kinase genes. A total of 11,292 genes were detected to be expressed in the AR42J cells. In the TLC-S group, a total of 1,124 genes were upregulated and 498 were downregulated. Through bioinformatics analysis, 22 differentially expressed protein kinase genes were found in the 1,124 differentially expressed genes, among which 19 genes were upregulated and 3 were downregulated (Table I). 
Table I. Differently expressed protein kinase genes in AR42J cells treated with taurolithocholic acid 3-sulfate (TLC-S).

\begin{tabular}{|c|c|c|c|c|}
\hline Gene name & Gene ID & Description & Mode of regulation & Fold change \\
\hline Sgk196 & 306549 & Sugen kinase 196 & Upregulated & 2.0006287 \\
\hline Map2k4 & 287398 & Mitogen-activated protein kinase kinase 4 & Upregulated & 2.0351782 \\
\hline Ptk2b & 50646 & Protein tyrosine kinase 2 beta & Upregulated & 2.3132236 \\
\hline Cdk1 & 54237 & Cyclin-dependent kinase 1 & Upregulated & 4.0139747 \\
\hline Lyn & 81515 & V-yes-1 Yamaguchi sarcoma viral related oncogene homolog & Upregulated & 3.2749004 \\
\hline Mapk14 & 81649 & Mitogen-activated protein kinase 14 & Upregulated & 2.2141974 \\
\hline Cdc42bpb & 113960 & CDC42 binding protein kinase beta (DMPK-like) & Upregulated & 2.1441314 \\
\hline Stk17b & 170904 & Serine/threonine kinase $17 \mathrm{~b}$ & Upregulated & 2.80085 \\
\hline Tesk2 & 170908 & Testis-specific kinase 2 & Upregulated & 2.557841 \\
\hline Rps6ka2 & 117269 & Ribosomal protein S6 kinase, $90 \mathrm{kDa}$, polypeptide 2 & Upregulated & 3.3565648 \\
\hline Sgk3 & 171498 & Serum/glucocorticoid regulated kinase 3 & Upregulated & 2.0300796 \\
\hline Ttk & 315852 & Tramtrack & Upregulated & 2.8380172 \\
\hline Melk & 362510 & Maternal embryonic leucine zipper kinase & Upregulated & 4.2185326 \\
\hline Mast2 & 313819 & Microtubule-associated serine/threonine kinase 2 & Upregulated & 2.1736362 \\
\hline Pak4 & 292756 & p21 protein $(\mathrm{Cdc} 42 / \mathrm{Rac})$-activated kinase 4 & Upregulated & 2.865645 \\
\hline Bub1 & 296137 & Budding uninhibited by benzimidazoles 1 homolog & Upregulated & 6.3756404 \\
\hline Irak3 & 314870 & Interleukin- 1 receptor-associated kinase 3 & Upregulated & 2.1035454 \\
\hline Prkca & 24680 & Protein kinase $\mathrm{C}$, alpha & Upregulated & 2.1087615 \\
\hline Mapk8 & 116554 & Mitogen-activated protein kinase 8 & Upregulated & 2.7886798 \\
\hline Fyn & 25150 & FYN oncogene related to SRC, FGR, YES & Downregulated & -2.3253782 \\
\hline Mst1 & 24566 & Macrophage stimulating 1 & Downregulated & -3.9783108 \\
\hline Ptk7 & 301242 & Protein tyrosine kinase 7 & Downregulated & -3.8359256 \\
\hline
\end{tabular}

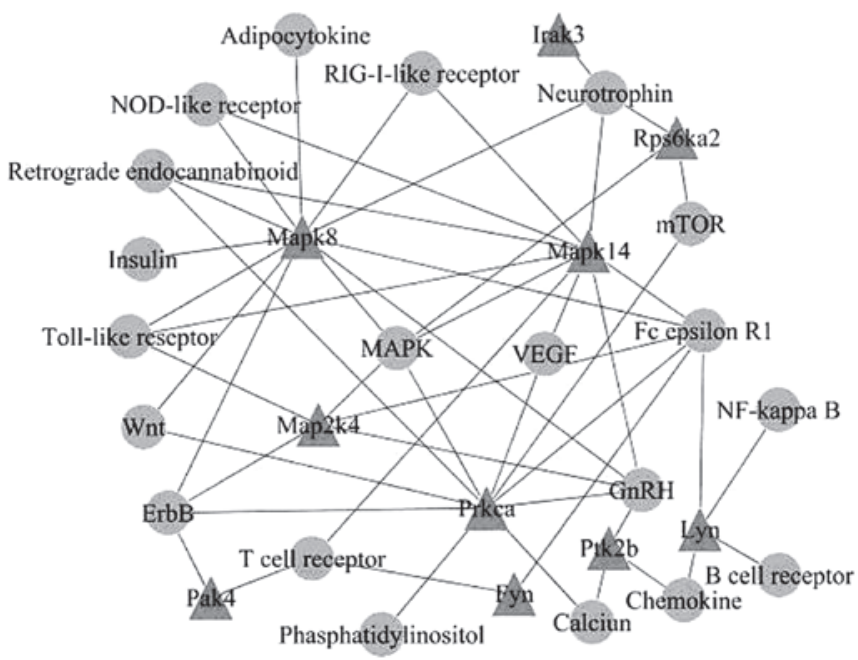

Figure 2. Network of interactions of the differentially expressed kinase genes. This network is composed of differentially expressed kinase genes and some related KEGG signaling pathways. Ellipse shape nodes refer to genes involved in signaling pathways (KEGG); triangle shape nodes refer to protein kinase genes.

Based on the KEGG database, kinase genes of the same KEGG pathways were connected to create a visualized network through signaling pathways, and 10 nodes of kinases were identified, which were mitogen-activated protein kinase (Mapk)8, Mapk14, Map2k4, interleukin-1 receptor-associated kinase 3

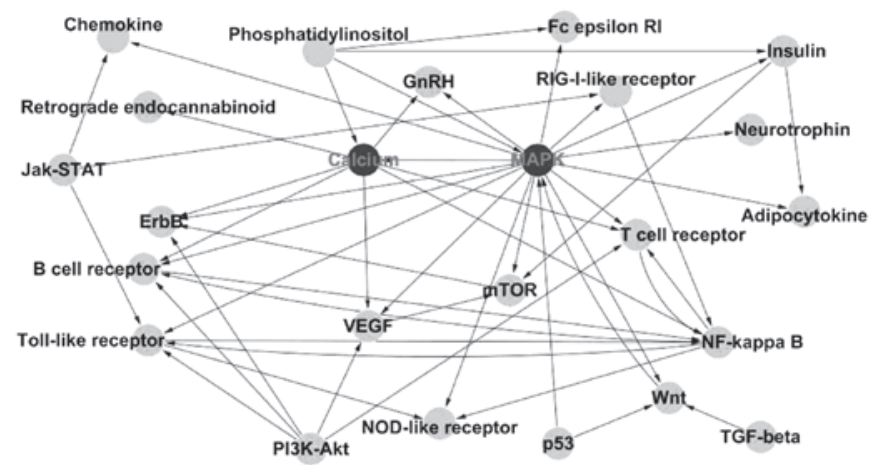

Figure 3. Network of interactions of signaling pathways. Each signaling pathway that showed differentially expressed kinase genes was imported into KEGG database, all the related pathways were searched, and the interactions were exhibited as a network. Each ellipse shape node refers to a signaling pathway.

(Irak3), ribosomal protein S6 kinase, $90 \mathrm{kDa}$, polypeptide 2 (Rps6ka2), protein kinase C, alpha (Prkca), v-yes-1 Yamaguchi sarcoma viral related oncogene homolog (Lyn), protein tyrosine kinase 2 beta (Ptk2b), p21 protein (Cdc42/Rac)-activated kinase 4 (Pak4) and FYN oncogene related to SRC, FGR, YES (Fyn) (Fig. 2).

Subsequently, the interactions between the signaling pathways were further analyzed and a network was created. MAPK and calcium signaling pathways were found to be located at the center of the network (Fig. 3). 
Table II. Regulation of trypsinogen activation based on literature mining.

\begin{tabular}{|c|c|c|c|}
\hline Regulators of trypsinogen activation & Journal & $\begin{array}{c}\text { Year of } \\
\text { publication }\end{array}$ & Authors/(Refs.) \\
\hline \multicolumn{4}{|l|}{ Cathepsins } \\
\hline Cathepsin B & $\mathrm{J}$ Clin Invest & 2010 & Reiser et al (6) \\
\hline Cathepsin B & Pancreatology & 2006 & Lindkvist et al (7) \\
\hline Cathepsin L & Gastroenterology & 2010 & Wartmann et al (8) \\
\hline \multicolumn{4}{|l|}{ Calcium signaling pathway } \\
\hline Calcineurin & Am J Physiol Gastrointest Liver Physiol & 2009 & Shah et al (9) \\
\hline Calcium release & Proc Natl Acad Sci USA & 2009 & Gerasimenko et al (10) \\
\hline Calpain & Int J Exp Pathol & 2009 & Weber et al (11) \\
\hline Ryanodine receptor regulation calcium & Proc Natl Acad Sci USA & 2005 & Husain et al (12) \\
\hline \multicolumn{4}{|l|}{ Protein kinase signaling pathway } \\
\hline $\mathrm{PKD} / \mathrm{PKD} 1$ & Am J Physiol Gastrointest Liver Physiol & 2011 & Throwe et al (13) \\
\hline Rho-kinase & Br J Pharmacol & 2011 & Awla et al (14) \\
\hline MAPK & Am J Physiol Gastrointest Liver Physiol & 2008 & Namkung et al (15) \\
\hline $\mathrm{PKC}$ & J Gastroenterol Hepatol & 2008 & Gorelick et al (16) \\
\hline Pgk1, Pgk2 & Pancreatology & 2012 & Li et al (17) \\
\hline \multicolumn{4}{|l|}{ Endothelins } \\
\hline Endothelins & Exp Toxicol Pathol & 2011 & Andrzejewska and Dlugosz (18) \\
\hline Endothelin-1 receptor antagonists & World J Gastroenterol & 2005 & Andrzejewska et al (19) \\
\hline \multicolumn{4}{|l|}{ Enterokinases } \\
\hline Enteropeptidase & Front Biosci & 2009 & Zheng et al (20) \\
\hline Enterokinase & Surgery & 2007 & Hartwig et al (21) \\
\hline
\end{tabular}

\section{Discussion}

Trypsinogen activation in pancreatic acinar cells is the initial factor involved in the development of all types of AP and causes the self-digestion of cells. Shortly after the induction of AP, immature secretory vesicles have been observed in the cytoplasm, and the activity of trypsin has been detected in this subcellular domain (4). However, the underlying mechanisms of trypsinogen activation remain to be elucidated. Several studies have shown that cathepsin and the increased calcium concentration in cells are essential for the activation of digestive enzymes (5). Over the past decade, the roles of protein kinases and the signaling pathways associated with trypsinogen activation have received increased attention (Table II).

Protein kinase, also known as protein phosphakinase, is a key regulator of numerous cellular functions. It changes the molecular structure by adding a phosphate group to substrate proteins in order to regulate protein activity, and it coordinates cellular processes by acting on different signaling pathways. Protein kinase is associated with almost every cellular function. Protein kinase, which is important in regulating cell behavior, is considered to be the target in the treatment for various diseases including tumors, diabetes, osteoporosis, phlogosis and oculopathy (22-24).

In the present study, the AR42J rat pancreatic acinar cell line was used to establish a model of trypsinogen activation in pancreatic cells following treatment with $200 \mu \mathrm{M}$ TLC-S (17). Total RNA from the AR42J cells was then extracted to perform whole-genome expression profile analysis, and 22 differentially expressed protein kinase genes were identified. Ten nodes of protein kinase genes were listed in a correlation diagram through analyzing KEGG pathways. MAPK and calcium signaling pathways were found to play an important role in trypsinogen activation.

The present study suggests that calcium signaling pathways play an important role in trypsinogen activation, and that Prkca and Ptk2b are associated with this signaling pathway. The association between AP and $\left[\mathrm{Ca}^{2+}\right]$ i in pancreatic acinar cells has been previously investigated (9). In 1995, Ward et al (25) hypothesized that calcium overload in acinar cells constitutes the main cause of AP development. Mooren et al (26) identified an increase in $\left[\mathrm{Ca}^{2+}\right] \mathrm{i}$ in acinar cells and an alteration of the normal calcium signals. The early use of BAPTA-AM, a calcium chelator, inhibits the above reaction. The abnormal release of calcium in cells and the aberrant activation of zymogen in acinar cells that cause self-digestion are known to be early events in the progression of AP (27). According to previous studies, the aberrant increase in $\left[\mathrm{Ca}^{2+}\right] \mathrm{i}$ in acinar cells has been shown to directly trigger trypsinogen activation and liquid cavity formation, since the treatment of pancreatic acinar cells with large doses of cholecystokinin (CCK, $10 \mathrm{nmol} / \mathrm{l})$ triggers trypsin activation of $\left[\mathrm{Ca}^{2+}\right] \mathrm{i}$-dependent top granules in acinar cells (28), and large doses of CCK cause changes in the calcium dependence of acinar cells in cellular morphology and the extensive replacement of zymogen granules by liquid cavity. Raraty et al (29) showed 
that the stably increased $\left[\mathrm{Ca}^{2+}\right] \mathrm{i}$ induces trypsinogen activation. Shah et al (9) observed that under the effect of FK506, a calcium/calmodulin-protein-dependent phosphatase inhibitor, the level of trypsinogen activation significantly decreased; a lower level of blood amylase and the inflammatory factor, interleukin (IL)-6, was also observed. The effect of FK506 was also investigated by Ozawa (30). Calcium/calmodulin calcineurin (PP2B) is controlled by many factors; however, the main mechanisms are the aberrant increase of $\left[\mathrm{Ca}^{2+}\right] \mathrm{i}$ in acinar cells and the alteration of calmodulin concentration (31). PP2B has also been shown to be the main target for the aberrant increase of $\left[\mathrm{Ca}^{2+}\right] \mathrm{i}$ in pancreatic acinar cells. The aberrant increase in $\left[\mathrm{Ca}^{2+}\right] \mathrm{i}$ in pancreatic acinar cells has been shown to activate PP2B, which is considered to be an important step in pathologic trypsinogen activation in pancreatic acinar cells.

MAPK, one of the serine/threonine protein kinases, presents in the majority of cells. It is one of the important signaling systems where eukaryotic cells transduce extracellular signals into cells to cause cellular reactions. Prior to activation, MAPK is located in the cytoplasm, and it enters the cell nucleus to activate the target gene following activation by phosphorylation upon stimulation. MAPK affects the gene transcription and regulation to influence the biological behavior of cells, such as cell proliferation, differentiation, transformation and apoptosis. The MAPK signaling pathway may be induced by pro-inflammatory molecules and stress, and is involved in most of the reactions of immunization and apoptosis caused by pro-inflammatory molecules (32). The present study identified the upregulated genes, Mapk14, Map2k4, Mapk8, Rps6ka2 and Prkca, that are associated with the MAPK signaling pathway, indicating that the MAPK signaling pathway plays an important role in trypsinogen activation.

There are 3 subgroups in the MAPK family, p38, ERK 1/2 and JNK. p38 has been proven to be specifically associated with the severity of AP and lung injury caused by severe AP. Thus, injuries in the lungs and part of the pancreas are reduced by inhibiting the activation of p38 (33). Certain studies have shown that during the inflammatory processes, oxidative stress and pro-inflammatory molecules are involved in the inflammatory cascade process by enabling the signaling transduction pathway, and that the activation of MAPK is of high importance during this process. Pereda et al (34) also demonstrated that the concurrent treatment of AP animal models with oxypurinol, a p38 inhibitor, and pentoxifyllin, an ERK 1/2 and JNK inhibitor, relieves inflammatory responses and reduces mortality. Additionally, the inhibition of the $\mathrm{p} 38$, ERK1/2 and JNK signaling pathways has been shown to have a less significant effect. The p38 signaling pathway specifically correlates with oxidative stress and the ERK 1/2 and JNK pathways are clearly associated with the expression of proinflammatory molecules. Since p38, ERK 1/2 and JNK have an independent effect on the process of AP development, concurrently inhibiting all 3 signaling pathways has a specific effect on relieving AP symptoms. It has been reported that oxidative stress causes MAPK activation and induces tumor necrosis factor (TNF)- $\alpha$ (35). TNF- $\alpha$ has also been suggested to be an initiator of cytokine cascade reaction during AP development. Williard et al (36) showed that p38 is involved in the activation of the pro-inflammatory nuclear transcription factor, nuclear factor (NF)- $\kappa \mathrm{B}$, to upregulate the expression of pro-inflamma- tory factors and aggregate AP. Samuel et al (37) demonstrated that the use of ERK 1/2 (PD98059), p38 (SB203580) and JNK (SP600125) inhibitors to suppress their activation in a biliary AP model, significantly reduced the expression of the pro-inflammatory cytokine in pancreatic tissue. Liu et al (38) supported this finding by comparing the serum and cytokine levels in the ascites of rats with severe and mild AP.

In conclusion, protein kinases are important in AP development, since they are associated with approximately every mechanism of AP development. As a result, protein kinases constitute potential drug targets for AP treatment. Gene therapeutic methods, such as gene transfection and RNA interference have proven to be successful in the treatment of AP in laboratory experiments; however, their use in clinical practice is still highly restricted. Targeting protein kinases as a form of therapy should be investigated in clinical trials, since a number of kinase inhibitors have been developed which may be used as a selective target. Therefore, further studies on the role of protein kinases in AP development are warranted, in order to develop a specific kinase inhibitor for the target therapy of AP.

\section{Acknowledgements}

This study was supported by the National Natural Science Foundation of China (30972907, 81070373). Financial support was also provided from the Postdoctoral Science-Research Developmental Foundation of Heilongjiang Province of China (LBH-Q11038).

\section{References}

1. Voronina S, Longbottom R, Sutton R, Petersen $\mathrm{OH}$ and Tepikin A: Bile acids induce calcium signals in mose pancreatic acinar cells: implications for bile-induced pancreatic pathology. J Physiol 540: 49-55, 2002.

2. Gerasimenko JV, Flowerdew SE, Voronina SG, et al: Bile acids induce $\mathrm{Ca}^{2+}$ release from both the endoplasmic reticulum and acidic intracellular calcium stores through activation of inositol trisphosphate receptors and ryanodine receptors. J Biol Chem 281: 40154-40163, 2006.

3. Perides G, Laukkarinen JM, Vassileva G and Steer ML: Biliary acute pancreatitis in mice is mediated by the G-protein-coupled cell surface bile acid receptor Gpbar1. Gastroenterology 138: 715-725, 2010 .

4. Thrower EC, Gorelick FS and Husain SZ: Molecular and cellular mechanisms of pancreatic injury. Curr Opin Gastroenterol 26: 484-489, 2010

5. Halangk W and Lerch MM: Early events in acute pancreatitis Clin Lab Med 25: 1-15, 2005.

6. Reiser J, Adair B and Reinheckel T: Specialized roles for cysteine cathepsins in health and disease. J Clin Invest 120: 3421-3431, 2010.

7. Lindkvist B, Fajardo I, Pejler G and Borgström A: Cathepsin B activates human trypsinogen 1 but not proelastase 2 or procarboxypeptidase B. Pancreatology 6: 224-231, 2006.

8. Wartmann T, Mayerle J, Kähne T, et al: Cathepsin L inactivates human trypsinogen, whereas cathepsin L-deletion reduces the severity of pancreatitis in mice. Gastroenterology 138: 726-737, 2010.

9. Shah AU, Sarwar A, Orabi AI, et al: Protease activation during in vivo pancreatitis is dependent on calcineurin activation. Am J Physiol Gastrointest Liver Physiol 297: G967-G973, 2009.

10. Gerasimenko JV,Lur G, Sherwood MW, et al: Pancreatic protease activation by alcohol metabolite depends on $\mathrm{Ca}^{2+}$ release via acid store IP3 receptors. Proc Natl Acad Sci USA 106: 10758-10763, 2009.

11. Weber H, Hühns S, Lüthen F and Jonas L: Calpain-mediated breakdown of cytoskeletal proteins contributes to cholecystokinin-induced damage of rat pancreatic acini. Int J Exp Pathol 90: 387-399, 2009 
12. Husain SZ, Prasad P, Grant WM, Kolodecik TR, Nathanson MH and Gorelick FS: The ryanodine receptor mediates early zymogen activation in pancreatitis. Proc Natl Acad Sci USA 102: 14386-14391, 2005.

13. Thrower EC, Yuan J, Usmani A, et al: A novel protein kinase D inhibitor attenuates early events of experimental pancreatitis in isolated rat acini. Am J Physiol Gastrointest Liver Physiol 300: G120-G129, 2011.

14. Awla D, Hartman H, Abdulla A, et al: Rho-kinase signalling regulates trypsinogen activation and tissue damage in severe acute pancreatitis. Br J Pharmacol 162: 648-658, 2011.

15. Namkung W, Yoon JS, Kim KH and Lee MG: PAR2 exerts local protection against acute pancreatitis via modulation of MAP kinase and MAP kinase phosphatase signaling. Am J Physiol Gastrointest Liver Physiol 295: G886-G894, 2008.

16. Gorelick F, Pandol S and Thrower E: Protein kinase C in the pancreatic acinar cell. J Gastroenterol Hepatol 23: S37-S41, 2008.

17. Li Z, Lu M, Chu J, Qiao X, Meng X, Sun B, Zhang W and Xue D: Early proteome analysis of rat pancreatic acinar AR42J cells treated with taurolithocholic acid 3-sulfate. Pancreatology 12: 248-256, 2012

18. Andrzejewska A and Dlugosz JW: Differential effects of endothelins on histological and ultrastructural changes and trypsinogen activation in the secretagogue-induced acute pancreatitis in rats. Exp Toxicol Pathol 63: 371-378, 2011.

19. Andrzejewska A, Dlugosz JW and Augustynowicz A: Effect of endothelin-1 receptor antagonists on histological and ultrastructural changes in the pancreas and trypsinogen activation in the early course of caerulein-induced acute pancreatitis in rats World J Gastroenterol 11: 1115-1121, 2005

20. Zheng XL, Kitamoto Y and Sadler JE: Enteropeptidase, a type II transmembrane serine protease. Front Biosci (Elite Ed) 1: 242-249, 2009

21. Hartwig W, Kolvenbach M, Hackert T, Fortunato F, Schneider L, Büchler MW and Werner J: Enterokinase induces severe necrosis and rapid mortality in cerulein pancreatitis: characterization of a novel noninvasive rat model of necro-hemorrhagic pancreatitis. Surgery 142: 327-336, 2007.

22. Morel M, Couturier J, Lafay-Chebassier C, Paccalin M and Page G: PKR, the double stranded RNA-dependent protein kinase as a critical target in Alzheimer's disease. J Cell Mol Med 13: 1476-1488, 2009

23. Kanda S, Miyata Y, Kanetake H and Smithgall TE: Non-receptor protein-tyrosine kinases as molecular targets for antiangiogenic therapy. Int J Mol Med 20: 113-121, 2007.

24. Katoh Y and Katoh M: FGFR2-related pathogenesis and FGFR2-targeted therapeutics (Review). Int J Mol Med 23 : 307-311, 2009
25. Ward JB, Petersen OH, Jenkins SA and Sutton R: Is an elevated concentration of acinar cytosolic free ionized calcium the trigger for acute pancreatitis? Lancet 346: 1016-1019, 1995.

26. Mooren FCh, Hlouschek V, Finkes T, et al: Early changes in pancreatic acinar cell calcium signaling after pancreatic duct obstruction. J Biol Chem 278: 9361-9369, 2003.

27. Frick TW: The role of calcium in acute pancreatitis. Surgery 152 : S157-S163, 2012

28. Kim JY, Kim KH, Lee JA, et al: Transporter-mediated bile acid uptake causes $\mathrm{Ca}^{2+}$-dependent cell death in rat pancreatic acinar cells. Gastroenterology 122: 1941-1953, 2002.

29. Raraty M, Ward J, Erdemil G, Vaillant C, Neoptolemos JP, Sutton R and Petersen OH: Calcium-dependent enzyme activation and vacuole formation in the apical granular region of pancreatic acinar cells. Proc Natl Acad Sci USA 97: 13126-13131, 2000.

30. Ozawa T: FK506 induces biphasic $\mathrm{Ca}^{2+}$ release from microsomal vesicles of rat pancreatic acinar cells. Int J Mol Med 18: 187-191, 2006.

31. Kessen U, Schaloske R, Aichem A and Mutzel R: Ca(2+)/calmodulin-independent activation of calcineurin from Dictyostelium by unsaturated long chain fatty acids. J Biol Chem 274: 37821-37826, 1999.

32. Conze D, Krahl T, Kennedy N, et al: c-Jun NH(2)-terminal kinase(JNK)1 and JNK2 have distinct roles in CD8(+) T cell activation. J Exp Med 195: 811-823, 2002.

33. Yang J, Murphy C, Denham W, Botchkina G, Tracey KJ and Norman J: Evidence of a central role for p38 map kinase induction of tumor necrosis factor alpha in pancreatitis-associated pulmonary injury. Surgery 126: 216-222, 1999.

34. Pereda J, Sabater L, Cassinello N, et al: Effect of simultaneous inhibition of TNF-alpha production and xanthine oxidase in experimental acute pancreatitis: the role of mitogen activated protein kinases. Ann Surg 240: 108-116, 2004.

35. Araki Y, Andoh A, Yokono T, et al: The free radical scavenger edaravone suppresses experimental closed duodenal loop-induced acute pancreatitis in rats. Int J Mol Med 12: 121-124, 2003.

36. Williard DE, Twait E, Yuan Z, Carter AB and Samuel I: Nuclear factor kappa B-dependent gene transcription in cholecystokinin and tumor necrosis factor-alpha-stimulated isolated acinar cells is regulated by p38 mitogen-activated protein kinase. Am J Surg 200: 283-290, 2010.

37. Samuel I, Zaheer S, Fisher RA and Zaheer A: Cholinergic receptor induction and JNK activation in acute pancreatitis. Am J Surg 186: 569-574, 2003.

38. Liu HS, Pan CE, Liu QG, Yang W and Liu XM: Effect of NF-kappaB and p38 MAPK in activated monocytes/macrophages on pro-inflammatory cytokines of rats with acute pancreatitis. World J Gastroenterol 9: 2513-2518, 2003. 